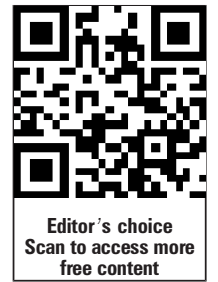

- Additional materials are published online only. To view these files please visit the journal online (http://dx.doi.org/ 10.1136/emermed-2012 201128)

${ }^{1}$ Belgian Red Cross-Flanders, Mechelen, Belgium ${ }^{2}$ Belgian Centre for Evidence-Based Medicine Leuven, Belgium

${ }^{3}$ Department of General Practice, Katholieke Universiteit Leuven, Leuven, Belgium ${ }^{4}$ Hanze University of Applied Sciences Groningen, Groningen, the Netherlands ${ }^{5}$ Research Unit Social and Preventive Health Care, Katholieke Universiteit Leuven, Leuven, Belgium

\section{Correspondence to} Dr Stijn Van de Velde, Belgian Centre for Evidence-Based Medicine, Kapucijnenvoer 33, block J, box 7001, Leuven 3000, Belgium; stijn.vandevelde@med. kuleuven be

Accepted 1 April 2012 Published Online First 5 May 2012

\title{
Can training improve laypersons helping behaviour in first aid? A randomised controlled deception trial
}

\author{
Stijn Van de Velde, ${ }^{1,2}$ Ann Roex, ${ }^{3}$ Karoline Vangronsveld, ${ }^{1}$ Lidewij Niezink, \\ Koen Van Praet, ${ }^{1}$ Annemie Heselmans, ${ }^{2}$ Peter Donceel, ${ }^{5}$ Philippe Vandekerckhove, ${ }^{1}$ \\ Dirk Ramaekers, ${ }^{2}$ Bert Aertgeerts ${ }^{2}$
}

\begin{abstract}
Background There is limited evidence indicating that laypersons trained in first aid provide better help, but do not help more often than untrained laypersons. This study investigated the effect of conventional first aid training versus conventional training plus supplementary training aimed at decreasing barriers to helping.

Methods The authors conducted a randomised controlled trial. After $24 \mathrm{~h}$ of conventional first aid training, the participants either attended an experimental lesson to reduce barriers to helping or followed a control lesson. The authors used a deception test to measure the time between the start of the unannounced simulated emergency and seeking help behaviour and the number of particular helping actions.
\end{abstract}

Results The authors randomised 72 participants to both groups. 22 participants were included in the analysis for the experimental group and 36 in the control group. The authors found no statistically or clinically significant differences for any of the outcome measures. The time until seeking help (geometrical mean and $95 \% \mathrm{Cl}$ ) was $55.5 \mathrm{~s}$ (42.9 to 72.0 ) in the experimental group and $56.5 \mathrm{~s}$ (43.0 to 74.3 ) in the control group. $57 \%$ of the participants asked a bystander to seek help, $40 \%$ left the victim to seek help themselves and 3\% did not seek any help.

Conclusion Supplementary training on dealing with barriers to helping did not alter the helping behaviour. The timing and appropriateness of the aid provided can be improved.

Trial registration The authors registered this trial at ClinicalTrials.gov as NCT00954161.

\section{INTRODUCTION}

Training laypersons in first aid is widely advocated to save lives. Substantial resources are devoted to voluntary or mandatory training efforts for the community at large or for specific target groups. ${ }^{1-5}$ To achieve this goal the training must improve knowledge, attitudes and skills, and the trainees must use these abilities appropriately in case of emergency.

Despite the general belief in the potential of first aid training, a systematic review showed that it does not always translate into helping behaviour. ${ }^{6}$ Three trials used deception experiments to evaluate the helping behaviour for emergencies after first aid training. ${ }^{7-9}$ The deception implied that the participants were unaware of any simulation and did not know that their helping behaviour was being evaluated. These trials showed that laypersons trained in first aid provided better help, ${ }^{7}$ but did not help more often than untrained laypersons. ${ }^{9}$ These trials also found that conventional first aid training was less effective than training supplemented by a training module that concentrated on the obstacles to action such as the presence of other bystanders and ambiguity about the emergency. ${ }^{8} 9$ The systematic review concluded that conventional first aid training improved competence but not performance, and that supplementary training on dealing with barriers to helping might enhance helping behaviour. ${ }^{6}$

The conclusions were only tentative because the evidence was of low quality and was limited to university student populations in the US. The review, in other words, highlights the need for further research into methods for training laypersons in overcoming these barriers. Therefore, this study aimed to investigate the effect of conventional first-aid training versus conventional training plus a supplementary educational module in a community-based randomised controlled trial because this provides the strongest design for questions about educational effectiveness. Our primary hypothesis was the time until seeking help is shorter among people trained with the supplementary intervention. Having the confidence to provide first aid may influence helping barriers. We therefore also measured the participants' self-efficacy beliefs and related these to the performance demonstrated in the deception. ${ }^{10}{ }^{11}$ While deception is a useful instrument to test first aid performance, this technique can create mistrust or can even harm the participants from high stress levels. ${ }^{12}$ For that reason this study also evaluated the impact of the deception on the participants.

\section{METHODS}

\section{Setting}

Belgian Red Cross-Flanders provides communitybased first aid training at almost 300 local branches, which are coordinated by its central services. Based on proximity to the headquarters of the Belgian Red Cross-Flanders, we selected six branches for participation in the trial.

\section{Selection of participants}

Red Cross staff recruited study participants from September 2009 until October 2010 during the first lesson of 10 first aid training courses organised by the selected branches. Participants had a minimum age of 18 years and were not allowed to follow additional first aid training outside the study. We excluded healthcare students and professionals 
from the study. Participants that completed the full study protocol received a gift coupon to the value of $€ 40$. The follow-up ended in January 2011.

\section{Study design}

This randomised controlled trial was approved by the University Hospitals Leuven Medical Ethics Committee. An investigator, who was not involved in the enrolment of study participants, used random number lists obtained from http://www.randomisation.com to allocate participants. The randomisation was done per branch, assuring an equal number of enrolled participants per group (if the number of participants was even).

\section{Interventions}

All participants initially received conventional first aid training. This was a 24-hour course, which covered first aid procedures for the most important emergencies including acute myocardial infarction. Teaching methods included lectures, skills practice on manikins or other participants, and exercises with simulated patients. No more than 20 participants were allowed per course. The participants were able to take a written and practical examination to assess competence, but this was not obligatory. Volunteer trainers gave the programme over 2 to 3 month periods. All trainers were trained in facilitation learning and assessment.

After this conventional first aid training, participants either attended the experimental training or the active control training. We informed participants that both lessons were newly developed and had to be evaluated. They received no information about the scope of these lessons until they started.

\section{Experimental training}

The experimental training module aimed to sensitise participants towards a helping reaction and to reduce barriers to helping. The training was based on Emergency Helping Behaviour Unit as presented by Hawks et $a l^{9}$ and on findings from research that documented the role of different factors on bystander helping attitudes or behaviour. ${ }^{13}{ }^{14}$ Among the barriers they document were presence of other bystanders, ambiguity about the emergency, fear of infection, being charged for calling an ambulance, being sued, or to increase the burden for the emergency medical systems without reason.

The instructional methods of the module included:

a) Group work and discussion on the likelihood of witnessing an emergency situation; personal experiences with emergencies; moral aspects of helping; feelings and perspectives of the victim and bystanders; barriers to helping, and how to deal with them.

b) Role play for specific situations where barriers to helping are present.

c) Formulation and writing down of personal helping goals.

In addition, the participants received a booklet containing information on barriers to helping and how to deal with them.

\section{Active control training}

The active control training focused on first aid for alcohol and drug incidents and was an extension of the conventional first aid training.

Both the experimental and control training lasted $2 \mathrm{~h}$ and took place 1 week after completion of the conventional training programme. The maximum number of participants per group was ten. One professional first aid trainer gave all the experimental and control lessons.

\section{Methods of measurement}

At baseline, we collected data on socio-demographic factors and scores on a multiple choice test measuring the knowledge about procedures for various first aid situations.

Directly after the experimental or control training, we measured first aid self-efficacy beliefs and satisfaction with selfdeveloped questionnaires that were tailored to the content and instructional methods. The self-efficacy scale contained six questions related to barriers to helping with the following structure: "I will do x (action) even if y (barrier)". ${ }^{11}$ The satisfaction questionnaire consisted of seven statements relating to novelty, comprehensibility, clarity, applicability of the information, teaching quality, group dynamics and overall satisfaction. The participants rated self-efficacy and satisfaction on a five and four point Likert scale respectively.

Within 1 month of the experimental or control training, we assessed each study participant individually during a deception test to evaluate the helping behaviour for a heart attack emergency (further details in the online supplementary file 1).

During the deception test, we assessed the primary outcome, that is, the time until seeking help. The time was measured between the moment that the simulation started and the moment that a person left the room to get help. This person could either be the study participant or an actor who had been instructed by the participant to get help. When no help-seeking action occurred after $3 \mathrm{~min}$, the test was aborted. Post hoc, we decided to explore how much time elapsed between (A) the start of the simulation and visual notice of the emergency by the participant: detection delay, (B) notice and any helping response: response delay, and $(\mathrm{C})$ notice and seeking help: help-seeking delay. A helping response was defined as either assessing the victim and seeking help, or providing direct help to the victim. Another outcome measured during the deception test was the number of particular helping actions. In order to be able to correctly interpret outcomes of this intervention, we also investigated the way participants had perceived the deception trial. Using 5-item Likert scales, the participants rated to one statement on credibility, to one statement on impact of deception and to ten statements on the state of positive and negative effect. For the state of mood, participants indicated how they felt in relation to five positive and five negative mood items: nervous, enthusiastic, confused, strong, ashamed, determined, anxious, proud, angry and inspired. The latter scale was based on the positive and negative affect schedule. ${ }^{15}$ All participants in the test were blinded for training group status.

Content experts and researchers in medical education, experimental psychology and social psychology evaluated the test scenario and questionnaires before the trial.

We estimated that we would need 25 participants in both the experimental and control group to detect a difference in mean delay time for seeking help of $30 \mathrm{~s}$ for a power of $80 \%$ at a $5 \%$ level of significance, a SD of $37 \mathrm{~s}$ and a control/experimental patient ratio of 1 . We periodically monitored whether the target number of participants for the analysis had been reached and then decided if new participants had to be enrolled.

\section{Primary data analysis}

For the time-related variables, we applied a logarithmic transformation (natural logarithm) to obtain a symmetric distribution of the model residuals. We reported the geometric mean values and $95 \%$ CIs in both groups after back-transforming (exponential function) to the original scale. Responses to items regarding self-efficacy, satisfaction and positive and negative mood were summed up per scale to yield composite scores. 
When a specific item was not answered, we imputed the mean individual score for the other completed items in the scale. The imputation was limited to maximum two ratings for each of the above-mentioned scales.

We used descriptive statistics for the baseline variables, rate of helping, various types of helping actions, credibility scores, the impact of event, the state of mood and satisfaction. We used Cronbach's $\alpha$ to evaluate the reliability of the self-efficacy and training satisfaction scale. To evaluate the time-related variables and the self-efficacy scores, we used a two-way analysis of variance. The first factor was the training branch; the second factor was the comparison group. Since the training branch was not an effect modifier, we reported the effect for all the training branches together. By calculating the Spearman's correlation coefficient, we evaluated the relationship between scores on the self-efficacy beliefs and the time-related variables. We initially planned to compare the rate of helping in both groups and to evaluate the relationship between scores on the self-efficacy beliefs and the rate of helping. Since all participants showed some type of helping reaction this was no longer relevant.

We analysed the data per protocol according to the group that the participants actually attended. Because suspicion about the deception test might influence helping behaviour, we excluded participants that reported credibility scores below four. An intention-to-treat analysis was not considered, since participants only switched groups when the time slot was not convenient for them.

A two-sided $\mathrm{p}<0.05$ was considered significant. We used PASW Statistics V.18 (SPSS) for the statistical analysis.

\section{RESULTS}

Figure 1 illustrates the participant flow in the trial. We randomised 72 participants to both the experimental and control group. Fifteen participants switched from group because the time slot of the allocated group was inconvenient for them. More participants dropped out in the experimental arm versus the controls. Time-related factors and bad weather with heavy snowfall were the main cause of this imbalance. We excluded two participants because they had prior knowledge of the deception. Nine participants were not included in the analysis because they did not find the deception sufficiently credible. One person who did not notice the emergency was also excluded.

Baseline data (table 1) are similar per allocated and per analysed group.
Figure 1 Flow of participants in the trial.

\section{Enrolment}


Table 1 Summary baseline statistics in allocated and attended groups

\begin{tabular}{lcccc}
\hline & $\begin{array}{l}\text { Experimental } \\
\text { group (n=72) }\end{array}$ & $\begin{array}{l}\text { Active control } \\
\text { group (n=72) }\end{array}$ & $\begin{array}{l}\text { Analysed in experimental } \\
\text { group (n=22) }\end{array}$ & $\begin{array}{l}\text { Analysed in active control } \\
\text { group (n=36) }\end{array}$ \\
\hline Sex (female) & $48(66.7 \%)$ & $48(66.7 \%)$ & $17(77.3 \%)$ & $25(69.4 \%)$ \\
Age (years) & $32.9(24.4-41.6)$ & $31.8(23.3-42.9)$ & $27.0(22.5-40.8)$ & $26.7(21.9-38.7)$ \\
Nationality (Belgian) & $69(95.8 \%)$ & $69(100 \%)$ & $21(95.5 \%)$ & $36(100 \%)$ \\
Education & & & & \\
$\quad$ Primary & $2(2.8 \%)$ & $0(0 \%)$ & $9(0 \%)$ & $1(2.8 \%)$ \\
$\quad$ Secondary & $35(48.6 \%)$ & $38(52.8 \%)$ & $9(40.9 \%)$ & $19(52.8 \%)$ \\
$\quad$ Higher & $35(48.6 \%)$ & $34(47.2 \%)$ & $13(59.1 \%)$ & $16(44.4 \%)$ \\
Profession & & & & \\
$\quad$ Employed & $51(70.8 \%)$ & $44(61.1 \%)$ & $12(54.5 \%)$ & $24(66.7 \%)$ \\
$\quad$ Retired/disabled & $4(5.6 \%)$ & $2(2.8 \%)$ & $2(9.1 \%)$ & $0(0 \%)$ \\
$\quad 1(1.4 \%)$ & $3(4.2 \%)$ & $1(4.5 \%)$ & $1(2.8 \%)$ \\
$\quad$ Housewife & $3(4.2 \%)$ & $7(9.7 \%)$ & $0(0 \%)$ & $4(11.1 \%)$ \\
$\quad$ Snemployed & $13(18.1 \%)$ & $16(22.2 \%)$ & $7(31.8 \%)$ & $7(19.4 \%)$ \\
Reladent & $38(52.8 \%)$ & $33(45.8 \%)$ & $12(54.5 \%)$ & $20(55.6 \%)$ \\
Red Cross volunteer (no) & $66(91.7 \%)$ & $63(87.5 \%)$ & $18(81.8 \%)$ & $34(94.4 \%)$ \\
Previous first aid training (no) & $49(68.1 \%)$ & $44(61.1 \%)$ & $13(59.1 \%)$ & $24(66.7 \%)$ \\
Experienced a life-threatening emergency (no) & $56(77.8 \%)$ & $49(68.1 \%)$ & $18(81.8 \%)$ & $27(75.0 \%)$ \\
Multiple choice test score (max. possible score=18) & $9(7-10)$ & $8(7-10)$ & $10(7-12)$ & $8(7-11)$ \\
\hline
\end{tabular}

Medians (01-03) or frequencies are reported.

We found no statistically or clinically significant differences between the experimental and control group for any of the measures. Table 2 shows the results for the time-related outcomes per analysed group. The ratio of the time until seeking help in the control group versus the experimental group was 1.02 (95\% CI 0.71 to 1.35$)$.

All participants showed some kind of helping reaction (table 3). Nearly all participants asked the victim what was wrong and took measures to obtain help. About 57\% ( $\mathrm{n}=33$ ) asked a bystander to seek help, $40 \%(n=23)$ left the victim to seek help themselves, and $3 \%(n=2)$ did not seek any help within the allocated $3 \mathrm{~min}$.

Most participants found the deception test rather impactful with an overall median (IOR) score of 4 (3 to 5) (possible range $=1$ to 5 ). We found substantially higher median (IOR) scores for the positive mood effect, 18.5 (15 to 20), than for the negative mood effect, 8 (6 to 10) (possible range 5 to -25 ).

Participants indicated a mean $(95 \% \mathrm{CI})$ self-efficacy score of 25.2 (23.9 to 26.6) in the experimental group and 24.4 (23.0 to 25.8 ) in the control group (Possible range 6 to -30 ; Cronbach's $\alpha 0.68)$. The self-efficacy score did not correlate with the timerelated outcomes. The experimental training group scored a median (IOR) of 21.5 (12 to 24 ) on the satisfaction scale (Possible range $=7$ to 28 ; Cronbach's $\alpha=0.94$ ) versus a median (IOR) of 18.5 (12 to 26 ) in the control group.

\section{LIMITATIONS}

In order to get the required sample size, we had to oversample substantially. Forty per cent of the enrolled participants dropped

Table 2 Geometrical means and 95\% Cls for time-related variables (seconds)

\begin{tabular}{lll}
\hline & $\begin{array}{l}\text { Experimental } \\
\text { group }(\mathbf{n}=\mathbf{2 2})\end{array}$ & $\begin{array}{l}\text { Active control } \\
\text { group }(\mathbf{n}=\mathbf{3 6})\end{array}$ \\
\hline $\begin{array}{l}\text { Time between start of } \\
\text { simulation and seeking help }\end{array}$ & $55.5(42.9-72.0)$ & $56.5(43.0-74.3)$ \\
$\begin{array}{l}\text { Detection delay } \\
\text { Response delay }\end{array}$ & $15.0(10.2-22.3)$ & $18.3(12.1-27.8)$ \\
Help-seeking delay & $4.0(2.2-7.3)$ & $4.8(2.6-9.1)$ \\
\hline
\end{tabular}

out before taking part in the experimental or control training. This high number is consistent with the drop-out rates that generally occur for this training. Considering the communitybased setting, the non-obligatory character of the training and the option to follow only part of it, the resulting adequately powered study can be seen as a strength. Although the perprotocol analysis involved groups of unequal size, that is, 22 and 36 subjects instead of the planned 25 subjects in each group, this deviation had only a minor impact on the actual power of the study. Based on the current sample there was $80 \%$ power to detect a difference of $59 \%$ between the control and the experimental groups. With a mean time equal to $55.5 \mathrm{~s}$ in the experimental group, this equals $32.8 \mathrm{~s}$, which is close to the difference of $30 \mathrm{~s}$ the study has been powered upon.

Although we found no difference in baseline data between those analysed and those who dropped out (results not reported), it is possible that unmeasured factors such as personality traits might have been important. We lost more participants in the experimental group than in the control group. This difference can be explained by the sign-up procedure for the deception experiment. In the first year of the trial, we asked the participants to sign up at the end of the control or experimental training. Since the active control training always preceded the experimental training, the control participants were able to select the most convenient timeslots on the

Table 3 Frequencies of types of helping reactions

\begin{tabular}{lcc}
\hline & $\begin{array}{c}\text { Experimental } \\
\text { group (n=22) }\end{array}$ & $\begin{array}{c}\text { Active control } \\
\text { group (n=36) }\end{array}$ \\
\hline $\begin{array}{l}\text { Any helping reaction (yes) } \\
\text { Asking victim what is wrong (yes) }\end{array}$ & $22(100 \%)$ & $36(100 \%)$ \\
$\begin{array}{l}\text { Seeking help } \\
\quad \text { By study participant }\end{array}$ & $91(95.5 \%)$ & $34(94.4 \%)$ \\
$\quad$ By bystander (at request of & $12(54.5 \%)$ & $14(38.9 \%)$ \\
study participant) & $1(58.3 \%)$ \\
$\quad$ By nobody & $1(4.5 \%)$ & $1(2.8 \%)$ \\
Asking about medication use (yes) & $10(45.5 \%)$ & $12(3.6 \%)$ \\
Attention to body position of victim (yes) & $8(36.4 \%)$ & $10(27.8 \%)$ \\
Telling the victim to stay calm (yes) & & \\
\hline
\end{tabular}


timetable. In the last part of the trial, we presented the timetable before the participants attended the experimental or control training. After this correction, the loss to follow-up became similar in both groups. Despite the high number of drop-outs, the comparability of the two groups remained intact.

A consequence of the high number of drop-outs is that the training groups were too small to give the experimental training according to the didactical plan. This might be a reason why the training did not make a difference.

Since we did not include a passive control group, we cannot evaluate whether similar or different results would have been obtained.

\section{DISCUSSION}

The experimental training on dealing with barriers to helping did not alter the time or rate of helping. The good news is that all the participants demonstrated some type of helping reaction and nearly all the participants sought help within 3 min after the start of the simulation. Ten per cent remained passive for more than $1 \mathrm{~min}$, which was a long time in the context of this study. Although the first aid training curriculum teaches participants to stay with the victim if bystanders are available to seek help, almost $40 \%$ left the victim to seek help themselves.

While our results regarding the time to helping response were similar to the findings of Hawks et al, we made some divergent observations regarding the rate of helping behaviour. It is remarkable that we found a $100 \%$ helping rate in our study versus $16 \%$ and $45 \%$ in the trials by Hawks. ${ }^{8}$ However, the trials differed in several ways. Participants in our communitybased study were European, older and included more women than the student-based US studies by Hawks. The diffusion of responsibility to help might have played a greater role in the studies by Hawks, with four to five passive bystanders versus two in our study. The emergency might have been more ambiguous in Hawks' studies, where the victim periodically grabbed for his heart and tried to catch his breath. In our study, the victim simulated the same signs but on a continuous basis, which might have made the emergency more explicit. It is clear that our scenario was not as challenging as the one by Hawks. It is not unlikely that the effect of the experimental helping behaviour training only becomes visible in more complex situations. The above also illustrates that it is difficult to extrapolate the findings from these scenarios to other emergency scenarios.

In the studies by Hawks, participants had 2 min to respond to the emergency, versus $3 \mathrm{~min}$ in our trial. After 2 min we already found a $93 \%$ helping rate. Almost half of the participants in Hawks 1992 study were moderately to extremely suspicious about the deception test. This proportion was considerably higher than in our study (13\%).

Another difference is that Hawks did not exclude these participants from the analysis. In our study, most participants were assessed within 2 weeks after the intervention versus within one or 2 months. The level of prior first aid training might also have an influence on the effectiveness of a helping behaviour training module. In our study, the level of prior first aid training was quite high $(24 \mathrm{~h})$. Unfortunately the amount of hours of previous training is not mentioned in the manuscripts by Hawks.

The first aid self-efficacy beliefs were on an average high, but did not predict the performance during the deception test. This corresponds with other studies which found that self-assessment of performance is often substantively flawed. ${ }^{16}$

A strength of this study is the evaluation of the effects of the deception test on the participants. Although deception is common in social-psychological research, this aspect is typically not evaluated. The satisfactory positive and negative mood scores, collected after the participants were told about the deception, provide support for their use in new trials on the helping behaviour of first aid trainees.

In this trial, we used deception as a tool to evaluate the effectiveness of a training intervention. It might also be interesting to evaluate the potential of the deception test as a teaching tool. ${ }^{17}$ It would be interesting to evaluate if the feedback given after the deception test has an effect on the helping behaviour of the participants.

A deception test could also be used as a reference standard to evaluate the predictive validity for actual helping behaviour of formal assessments leading to a certificate of first aid competence.

\section{CONCLUSIONS}

This trial found no difference in time or rate of helping between conventional first aid training and conventional training plus a supplementary training to reduce barriers to helping. Although the high loss to follow-up was an important limitation of this study, we believe this study was a methodologically sound randomised controlled trial. This study contributes to the limited experimental research into the potential of training to reduce barriers to helping. Despite the unexpected high rates of seeking help or providing help in general, this study shows that there is a need to increase the timing and appropriateness of the aid provided. With regard to first aid self-efficacy beliefs, we conclude that these were not useful to predict performance during a real-life emergency. This study also gives insights into effects of deception tests on the participants and its potential in further research on first aid.

Acknowledgements The authors thank the Laerdal Foundation for providing funds for this study. The authors are grateful to John Collins, Annick Dermine, Richard Grol and Vera Hoorens for their critical reflections on the study protocol. They thank Steffen Fieuws for his advice on the design and elaboration of the statistical analysis. They would also like to thank the volunteers, Medical Committee, and staff of the Belgian Red Cross-Flanders for their help in implementing the trial.

Contributors SVdV, BA and DR conceived and designed the study. SVdV wrote the protocol. BA and DR gave methodological advice. AR provided a medical education perspective. KV provided an experimental clinical and health psychology perspective LN provided a social psychology perspective. KVP provided a psycho-social support perspective. PV, PD, AH provided general advice on the study. BA was the guarantor of the study.

Funding This study was co-funded by the Belgian Red Cross-Flanders and the Laerdal Foundation for Acute Medicine. The Laerdal Foundation was not involved in any part of the study.

Competing interests PV, SVDV, KVP are employed and KV volunteers in the Belgian Red Cross-Flanders which provides training in first aid and which received funding for this study from the Laerdal Foundation for Acute Medicine; AH, AR, BA, DR, LN, PD have no financial or non-financial interests that may be relevant to the submitted work.

Patient consent No patient images are being used. Custom patient consent forms have been used in the study.

Ethics approval The ethics approval was provided by University Hospitals Leuven Medical Ethics Committee.

Provenance and peer review Not commissioned; externally peer reviewed.

\section{REFERENCES}

1. Bollig G, Myklebust AG, Ostringen K. Effects of first aid training in the kindergarten a pilot study. Scand J Trauma Resusc Emerg Med 2011:19:13.

2. Anderson GS, Gaetz M, Masse J. First aid skill retention of first responders within the workplace. Scand J Trauma Resusc Emerg Med 2011;19:11.

3. Adelborg K, Thim T, Secher N, et al. Benefits and shortcomings of mandatory first aid and basic life support courses for learner drivers. Resuscitation 2011;82:614-17. 
4. Blewer AL, Leary $\mathrm{M}$, Decker CS, et al. Cardiopulmonary resuscitation training of family members before hospital discharge using video self-instruction: a feasibility trial. J Hosp Med 2011;6:428-32.

5. Auf der Heide $\mathbf{E}$. The importance of evidence-based disaster planning. Ann Emerg Med 2006;47:34-49.

6. Van de Velde S, Heselmans A, Roex A, et al. Effectiveness of Nonresuscitative first aid training in laypersons: a systematic review. Ann Emerg Med 2009;54:447-57.

7. Shotland RL, Heinold WD. Bystander response to arterial bleeding: helping skills, the decision-making process, and differentiating the helping response. J Pers Soc Psychol 1985; 49:347-56.

8. Hawks SR, Peck SL, Vail-Smith K. An educational test of health behavior models in relation to emergency helping. Health Psychol 1992;11:396-402.

9. Hawks SR, Egan M. The impact of three different first aid curricula on emergency helping among college students. J Health Educ 1998;29:289-93.

10. van der Bijl JJ, Shortridge-Baggett $L M$. The theory and measurement of the self-efficacy construct. Sch Inq Nurs Pract 2001;15:189-207.
11. Luszczynska A, Gutiérrez-Dona B, Schwarzer R. General self-efficacy in various domains of human functioning: evidence from five countries. Int J Psychol 2005:40:80-9.

12. Pittenger DJ. Deception in research: distinctions and solutions from the perspective of utilitarianism. Ethics Behav 2002;12:117-42.

13. Rowe BH, Shuster M, Zambon S, et al. Preparation, attitudes and behaviour in nonhospital cardiac emergencies: evaluating a community's readiness to act. Can $J$ Cardiol 1998;14:371-7.

14. Smith KL, Cameron PA, Meyer AD, et al. Is the public equipped to act in out of hospital cardiac emergencies? Emerg Med J 2003;20:85-7.

15. Watson D, Clark LA, Tellegen A. Development and validation of brief measures of positive and negative affect: the PANAS scales. J Pers Soc Psychol 1988;54:1063-70.

16. Dunning D, Heath C, Suls JM. Flawed self-assessment: implications for health, education, and the workplace. Psychol Sci Publ Interest 2004;5:69-106.

17. Elman D, Hooks R, Tabak D, et al. The effectiveness of unannounced standardised patients in the clinical setting as a teaching intervention. Med Educ 2004;38:969-73. 